\title{
A minimum physical distance delivery protocol based on ZigBee in smart grid
}

Jiasong Mu

\begin{abstract}
ZigBee provides a simple and reliable solution for the advanced measuring infrastructures. However, the current routing algorithms cannot fully satisfy the requirements of the application, and the characteristics of the node deployment and the data flows should be more considered. In this paper, we propose a minimum physical distance (MPD) delivery protocol based on the ZigBee specification in the smart grid to optimize the transmission of the monitoring and command packets which are from or to the ZigBee coordinator (ZC). The physical depth, which is introduced to indicate the least hops to the ZC, and the transmission paths are decided based on the neighbour table information. The simulation results show that the MPD could improve the performance of the monitoring and controlling packet transmission, it provided high reliability and short paths, the bits sent by the devices except the coordinator were reduced and the end-to-end delay was also shortened.
\end{abstract}

Keywords: ZigBee; Delivery protocol; Physical depth; Neighbour table

\section{Introduction}

Smart grid is characterized by two-way flow of power in electrical network, and information in communication network to increase energy efficiency, transition to renewable energy sources, reduce greenhouse gas emissions, and build a sustainable economy that ensures prosperity for current and future generations [1-3]. The real-time communication ability of the smart grid will enable utilities to optimize and modernize the power grid in order to realize its full potential [4]. The communication network takes charge of the collection and analysis of real-time data, along with the control of electrical loads for energy reduction and demand response [5]. Advanced metering infrastructure (AMI) is the technology of automatically collecting data from energy metering devices and transferring that data to a central database by communication technology for remote control and analyzing. It is the totality of systems and networks for measuring, collecting, storing, analyzing, and using energy usage data. AMI will link consumers and power utilities together and provide foundation for future distribution automation and other smart grid functionalities [6]. Based on these functions, the nodes in AMI are always irregularly distributed and

Correspondence: mujiasong@aliyun.com

College of Electronic and Communication Engineering, Tianjin Normal University, Tianjin 300387, China the communication is low data rate and short range. In the recent report on National Institute of Standard and Technology (NIST) framework and roadmap for smart grid interoperability standards, several wireless communication technologies are identified for smart grid. For examples, ZigBee and the ZigBee Smart Energy Profile (SEP) have been defined as the one of the communication standards for use in the customer premise network domain (including AMI) of the smart grid [7].

ZigBee technology is characterized by low cost, low power, low data rate, and simplicity [8]. These features, along with its operating over unlicensed spectrum and being a standardized protocol based on IEEE 802.15.4 standards, facilitate easy network deployment and implementation, and make it the most suitable wireless technology for smart grid applications [9]. It has also been selected by a large number of utilities as the communications platform of choice for their smart metering devices as it provides a standardized platform for exchanging data between utilities and smart metering devices and appliances located on customer premises [10].

ZigBee uses a mixed routing mechanism combined with HRP (hierarchical tree routing) and Z-AODV (ZigBee ad-hoc on demand distance vector) [11]. HRP is based on the address distribution and provides a simple and reliable measure for data transmission, though it is not always

\section{穴}


efficient and robust. For Z-AODV, each node may initiate routing discovery when necessary; a global shortest path between the source and destination is obtained during the process, and the data frame was sent along the route. However, since the HRP and Z-AODV are designed for different topologies, their benefits are alternative. Moreover, ZigBee devices have limited processing capabilities, storage, power supplies, and communication bandwidth. They may also move about randomly, which results in topology changes of the network. These constraints make it very difficult to find proper routing mechanisms that ensure high network throughput in different environments [12]. For that reason, current network formation and routing protocols described in the ZigBee specification cannot fully address power consumption issues $[13,14]$. The deployment in smart grid is newly presented and the characteristics of application need to be more considered $[15,16]$.

The diagram of AODV is shown in Figure 1. One can see at least two separate modes: route discovery/maintenance (phases I and II) and the actual forwarding of application packets (phase III), with the first mode involving special traffic that does not directly originate at the network's application [17]. The routing request is always flooding by rebroadcasting while the data is unicast transmitted. The prevailing wisdom regarding the organization of wireless networks assumes point-to-point communication, whereby each node forwarding the packet on its way to the destination sends it to a specific neighbour [18].Note that the benefits of unicast mechanism tend to be questionable [19] and much more so in sensor networks, where packets tend to be very short [20]. Firstly, the action of announcing the transmission with the handshake may take more bandwidth than the actual transmission; so the probability of damage to an unannounced transmission is in fact lower than that to the announcement [21]. Secondly, the neighbour identifier requires room in the packet header and thus incurs extra framing, which significantly inflates the otherwise short packet. Therefore, the mechanism in Figure 1 has two major disadvantages: one is the higher latency caused by routing discovery; the other one is that the bandwidth cost in the first two phases and the unicast in phase III have no more gains for short data packet delivery. Owing to the open nature in the wireless channel, the unicast is essentially a broadcasting that only a specified device would respond by identifying address information. If other parameters which are able to control the data flow and restraint the flooding can be found, we can design a proper delivery protocol to overcome the mentioned defects and achieve better performance in data transmission.

Considering the regular data flows in AMI, they can be classified into two categories. One is the monitoring and controlling frames from or to the sink node, which is the ZigBee coordinator (ZC) in the ZigBee network. The other is the general communication between any other devices except the $\mathrm{ZC}$. For the monitoring and controlling communication, as the $\mathrm{ZC}$ is the root of tree structure, these packets are transmitted along the hierarchical paths, where the depths of nodes are strictly monotonic (increasing for command and decreasing for monitoring frames). The depth is defined as the hop counts in the HRP, and it indicates the topological distance to the ZC. Nevertheless, the parameter that implies the spatial distance is needed to find the best route. In our work, we introduce the physical depth (PD), which is the minimum hop counts to the sink, to indicate the physical distance from a local device to the ZC. Since all the one-hop neighbour node information is required to be stored by each device according to the ZigBee specification, the physical distance can be easily updated by diffusion. On that basis, the transmission of the command and monitoring packets could be optimized by the principle of seeking the receiver with the least physical depth in broadcasting transmission. In this way, the monitoring and command frames can be transmitted in the global shortest path with neither the handshake nor the routing discovery.

The rest of this paper is organized as follows: The related works are reviewed in section 2 . Section 3 briefly introduces the ZigBee specification, and the minimum physical distance broadcasting algorithm is proposed in section 4 . In section 5 , simulation results are presented. Finally, the conclusion is shown in section 6.

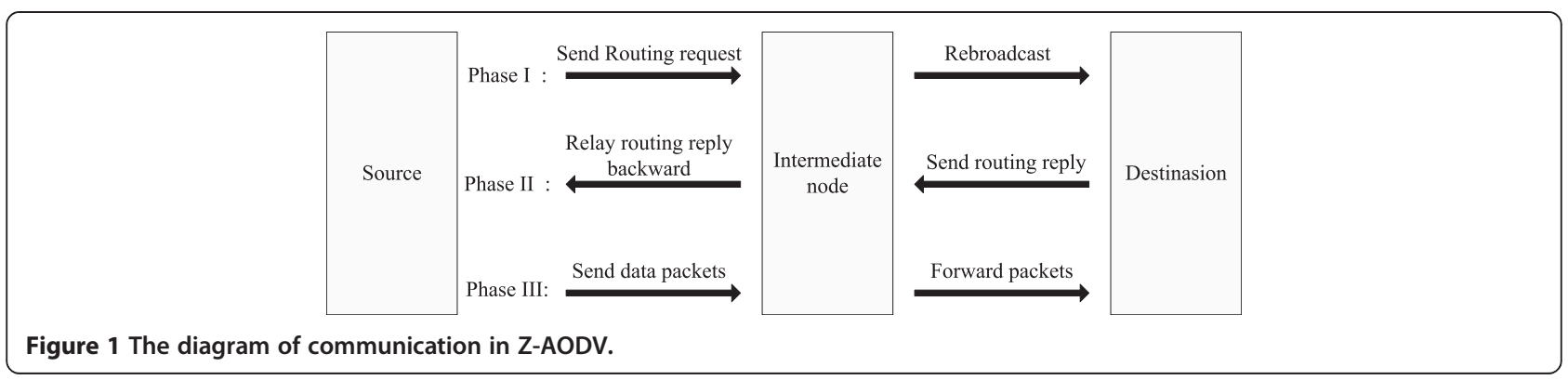




\section{Related works}

Recently, the ZigBee network has been proposed in different research areas in power systems. The IEEE 802.15.4 was used to construct a wireless, non-intrusive, intelligent, and low-cost energy management system $[22,23]$. The motor terminal voltages and current information are sent through a WSN for energy evaluation and condition monitoring. Some progress in system design, network deployment, and data compression have been made by integrating ZigBee networks into Advanced Metering Infrastructure (AMI), fault monitoring, and the like [24,25]. Typically, a Power Monitoring Module (PMM) is proposed in [26], which integrates ZigBee and digital signal processing techniques for wireless communication and real-time power parameters computation. The proposed system is stand-alone and communicates wirelessly with outside systems hence can be used in monitoring different points in the power system.

The MPD proposed in this paper is based on the hierarchical structure, in which the HRP is commonly used. For the HRP, paper [27] gives a modified tree routing mechanism with the introduction of neighbour table. The transmission cost (e.g. hops) via each neighbour device is estimated and compared to improve the routing path. It has a better performance with less power consumption per packet transfer and a long life cycle. But this algorithm is based on the two-hop neighbour information and it may lead to severe energy and memory overhead in ZigBee networks. Studies [28-30] propose several similar routing algorithms for hierarchical topology. The information in neighbour tables was used to get the shorter paths. Based on the Distributed Address Allocation Mechanism (DAAM), if the addresses of source and destination nodes are given, the hierarchical route can be decided without any other information. The local device may calculate the hierarchical hop counts for every device in the neighbourhood and choose the one with the least hops as the next hop node. This mechanism can only grant the best hierarchical path, while our work may find the route with the shortest physical distance. Moreover, the MPD utilizes the broadcasting transmission for better performance, the receiving node but not the sending one may decide whether it should retransmit the packet. The most relevant work is in [31], a tiny and efficient protocol is proposed based on broadcasting. The algorithm may provide less frame bits and shorter delay. Meanwhile, the protocol is also proved reliable. The simulation results show that the method has a high-enough packet delivery fraction, in the static network, this ratio was no less than 95\%. However, the protocol is proposed in common wireless networks and the improvement need further evaluation due to the undetermined MAC layer standards. Our scheme fits for the ZigBee specification, the neighbour information could be used for further optimization. And we compare our method with other popular routing algorithms in the ZigBee network to evaluate the performance objectively.

\section{ZigBee specification and routing methods \\ 3.1 Link quality indication}

ZigBee devices support the function of testing the link quality indication (LQI) measurement every time they receive a frame. The LQI measurement is a characterization of the strength and/or quality of a received packet. The measurement may be implemented using receiver energy detection (ED), a signal-to-noise ratio estimation, or a combination of these methods. The use of the LQI result by the network or application layers is not specified in IEEE 802.15.4 standard.

The LQI measurement shall be performed for each received packet, and the result shall be reported to the MAC sublayer. The minimum and maximum LQI values $(0 \times 00$ and $0 \times \mathrm{ff})$ should be associated with the lowest and highest quality IEEE 802.15.4 signals detectable by the receiver, and link quality (LQ) values in between is uniformly distributed between these two limits. The LQI information of every single received packet can be simply acquired according to the standard with no more extra calculation and communication [8].

\subsection{Address allocation and HRP}

In ZigBee specification, it is recommended to use the DAAM for address assignment to form the tree structure. The parameter $\mathrm{Cm}$ represents the largest number of children nodes, $R m$ means the number of children nodes which can be a router, and $L m$ decides the maximum depth in the network. And for the same network, different nodes usually have constant $\mathrm{Cm}$ and $\mathrm{Rm}$. Every potential parent is provided with a finite sub-block of the address space, which is used to assign network addresses to its children. Given $C m, L m$, and $R m$, we can compute the function $C \operatorname{skip}(d)$ as the size of the address sub-block distributed by each parent at depth $d$ as (1) [8].

$$
C \operatorname{skip}(d)=\left\{\begin{array}{cl}
0, & R m=0, \\
1+C m \times(L m-d-1), & R m=1, \\
\left(1+C m-R m-C m \times R^{L m-d-1}\right) /(1-R m), R m>1 .
\end{array}\right.
$$

The network addresses $A_{d+1, \mathrm{r} n}$ and $A_{d+1, \mathrm{e} l}$ shall be assigned to the $n$th router child and $l$ th end device child at depth $d+1$ in a sequential manner, respectively, as shown in (2):

$$
\begin{gathered}
A_{d+1, \mathrm{r} n}=A_{\text {parent }}+C \operatorname{skip}(d) \times(n-1)+1 \\
A_{d+1, \mathrm{e} l}=A_{\text {parent }}+C \operatorname{skip}(d) \times R_{m}+l
\end{gathered}
$$


where $A_{\text {parent }}$ represents the address of the parent and $1 \leq n \leq R m$.

The hierarchical topology in the ZigBee network is based on the DAAM. In this tree-shaped structure, if the destination address is in the address space that a node is managing, the node forwards the packet to one of its child nodes. Otherwise, it forwards the packet to its parent.

\subsection{Neighbour table}

Each ZigBee device maintains a neighbour table which has all its neighbours' information in the one-hop transmission range. The contents for a neighbour entry are the network's personal area network (PAN) identifier; node's extended address, network address, device type, relationship, LQI, etc. Optionally, additional information such as the depth can be included. Entries in the table are created when the node joins an existing network. Conversely, the neighbour entry is removed when the neighbour node leaves the network. Since the information on the neighbour table is updated every time a device receives any frame from the some neighbour node, the information of the neighbour table can be said to be up-to-date all the time.

\section{Minimum physical distance broadcasting algorithm}

In the smart grid application, the monitoring and command packets include the electricity consumption information gathering from the appliances and the instructions controlling the equipment. These packets are either collected or sent by the smart meter. In other words, the communication has a definite terminal, the ZC. The depth is defined as the transmission hops in the HRP. It indicates the topology distance between the local device and the ZC. This information is not helpful to find the global shortest path since the hierarchical topology limits the transmission to parent-child links. We introduce the concept of the physical depth (PD), which is defined as the minimum hop counts to the $\mathrm{ZC}$. So the PD is able to indicate the spatial distance to some extents.

Thus, the $\mathrm{ZC}$ is the only device with the PD 0 in the network. For a device in the neighbourhood of the $\mathrm{ZC}$, its PD may be set to 1 since it may find the entry of the $\mathrm{ZC}$ in its neighbour table. If the PD information is required in the neighbour table, all the nodes within the transmission range of the PD 1 devices may have PD 2; the PD of any device can be recursively decided. The principle can be simply stated as follows: The PD value of a certain node is one plus the minimum $\mathrm{PD}$ value in its neighbour table.

However, there is no such content in the neighbour table in the ZigBee specification. Fortunately, the node depth is an optional term. Moreover, if the network address is assigned as the DAAM and the network address is given, the depth of the node can be calculated as the following pseudo-codes [29]:

Pseudo-codes: Algorithm of deciding the topology depth for node X

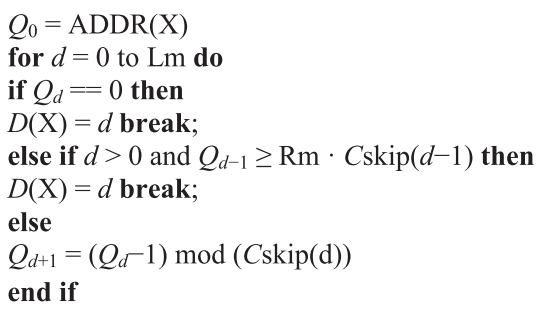

where $Q_{d}$ is introduced to determine whether node $\mathrm{X}$ is a router node or an end device at depth $\mathrm{d}$. One can see that the depth information is redundant in this case. Therefore, it can be replaced by the PD. On that basis, the delivery protocol is investigated.

In the MPD, we focus on the following parameters in the transmission. The address of the other terminal except the ZC, $S / D$ add (this device is also called S/D node in the following for convenience); the frame type, $M / C$ (1 for monitoring frame and 0 for command); the sequence number, $S N$; the retired times, $R T$; the PD of the $S / D$ node, $P D s$; the remaining hops to the destination, PDr; a flag, fOpt, indicates whether this frame is allowed to be rebroadcasted by other nodes which cannot find the routing table entries corresponding to the $S / D$ add ( 1 for True and 0 for false). All the variables above are included in the frame header. With the array of $[S / D$, $M / C, S N, R T]$, which we called broadcasting frame identity array (BFIA), the frame can be uniquely recognized. And the other parameters, $P D s, P D r$, and fOpt are used to control the transmission.

Our algorithm is based on an assumption that all the transmission links are half-duplex and symmetric. On the first attempt to communicate with the $\mathrm{ZC}$ for a certain node, suppose it is a monitoring frame (the principle is the same for the controlling one), the $S / D$ add is set the address of this device, $M / C$ is $1, S N$ is one plus the $S N$ value in the last monitoring packets transmission, $R T$ and $P D$ s depends on the real situation, $P D r$ is equal to the $P D s$ minus 1 , and $f$ Opt has to be 1 . And the packet is broadcasted to its neighbourhood by the S/D device.

Each node that receives this packet may compare the $P D r$ value with its own PD, if the PDr is smaller, the frame is abandoned. Otherwise, the device may rebroadcast the packet. Meanwhile, the $P D r$ value is decremented by 1 and an entry containing the $S / D$ add and the PDr is built in the routing table. The sending node is asked to monitor the channel, if some packet with the same BFIA and the $P D r$ is received, it may regard the packets as the transmission acknowledgement and reserve the corresponding 
routing entry, otherwise, the entry is deleted after a preset expiration time. Based on this mechanism, the frame is forwarded to the $\mathrm{ZC}$ and the path must be the global shortest.

When a packet is successfully delivered to the $\mathrm{ZC}$, all the nodes in the transmission path may update their routing tables. The entry should at least includes the $S / D$ add, PDs and PDr. For the later communication between this device and the $\mathrm{ZC}$, the fOpt could be set to 0 , and the nodes only have to check the existence of the corresponding routing table entry to decide whether it should rebroadcast this packet or abandon it. For the monitoring packets, the $P D \mathrm{r}$ is the $\mathrm{PD}$ value of the local device, while it is equal to (PDs-PD) for the controlling ones.

However, if more than one receiving nodes have the same minimum PD in one delivery, it leads to the multipath problem, as shown in Figure 2. Node 2 sends a packet with the $P D$ r set 3 , both the node $\mathrm{D}$ and $\mathrm{F}$ may rebroadcast this packet. Since all the candidates of next hop, including the LQI information, are in the neighbour table, a device may be aware of the branches before the transmission by checking whether there are more than 1 neighbours having the same minimum PD. If so, the sending device is asked to specify the network address of the receiving node. Such packets seem like the ones in the traditional unicast mechanism, but they have more simple frame header, e.g. the address of sending device is not necessary. Moreover, the address specifying only happens when the parameter fOpt is 1 (always in the first communication attempt), and the separate acknowledgement is removed to save the bandwidth.

The assumption of symmetric links can be roughly satisfied in most cases for the deployed nodes except the ZC. Owing to the sufficient power supply and capability of using more complicated peripherals, its transmitting range can be larger than the receiving one. This may lead to the routing errors and link failures. In our algorithm, all the nodes that could hear from the $\mathrm{ZC}$ are required to send a simple inquiry with its network address. The particular frame can be only responded by the ZC. If a node receives the response within a preset expire time, its $\mathrm{PD}$ is decided as 1 . Otherwise, it may ignored the neighbour table entry corresponding to the $\mathrm{ZC}$ and decide its depth based on the least PD value of the other neighbours. Note that for the controlling packets transmission, it is possible to have one or more less hops comparing with the monitoring ones since the $\mathrm{ZC}$ has the capability of sending message directly to the high PD devices. On that case, the $\mathrm{ZC}$ cannot receive the rebroadcasting to confirm the transmission. While the PD 1 node in the monitoring link may find the PDr value is less than expected. It is required to announce the situation. So the $\mathrm{ZC}$ is aware of the asymmetric link, and it may repeat the packets for bits error control. This mechanism not only solves the asymmetric link problem. The nodes close to the $\mathrm{ZC}$ tend to take part in the communication more frequently, thus they may consume the batteries faster. This framework can also reduce the energy consumption for low PD devices.

An example of the MPD routing is shown in Figure 2. We can see that the node 1 could send frames via node $\mathrm{C}$ to the $\mathrm{ZC}$, the hop counts is 2 , reduced by 1 compared with the value in the HRP. For the node 2, the transmission path is also shortened. The link can be uniquely determined since node 2 can specify the address of the next hop device when it finds both the node $\mathrm{D}$ and $\mathrm{F}$ have the same minimum $\mathrm{PD}$ in its neighbour table. And the links not chosen can be used as a backup for link failures. The asymmetric links close to the $\mathrm{ZC}$ is illustrated. When the $\mathrm{ZC}$ tries to send a controlling frame to the node 2 , it may directly send the packet whose $P D r$ is 1 . The length of downlink can be further

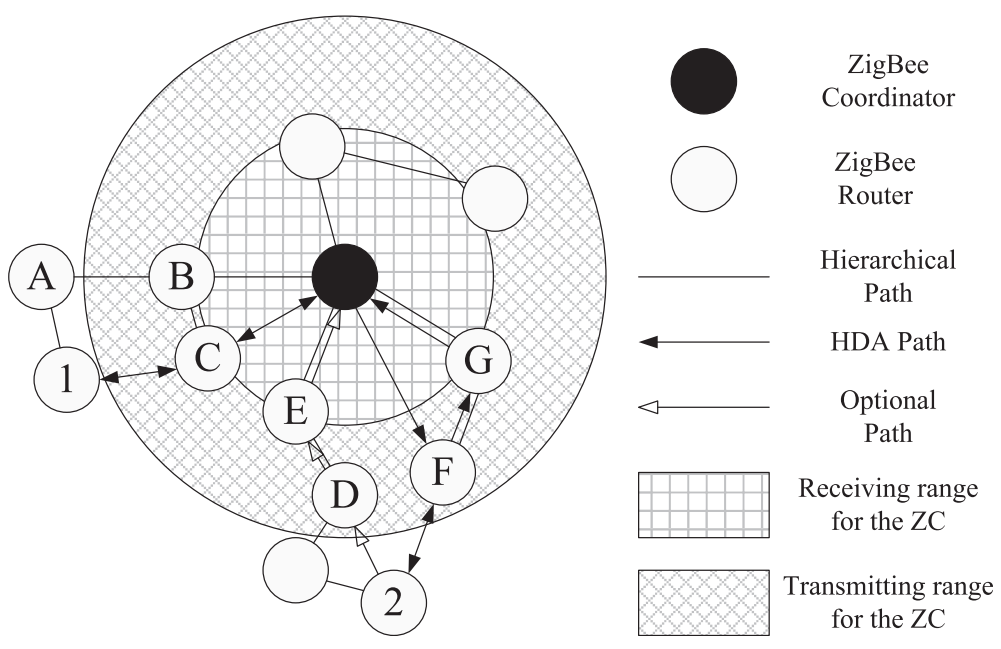

Figure 2 An example of the minimum physical distance broadcasting algorithm. 


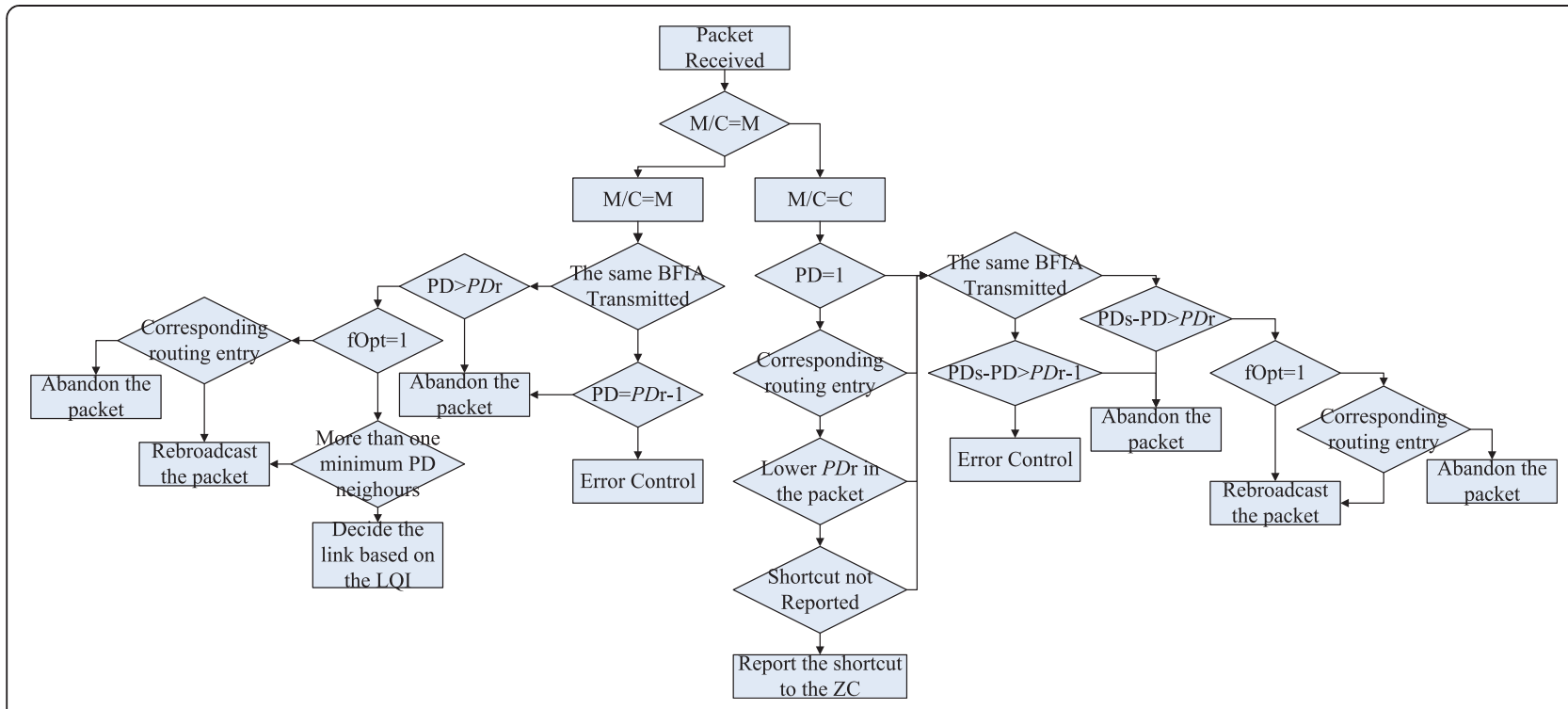

Figure 3 The diagram of the minimum physical distance broadcasting algorithm.

shortened to 2 . And the diagram of the node behaviours is shown in Figure 3.

\section{Simulations}

The performance of the proposed minimum physical depth delivery protocol is discussed in this section. The simulation was implemented by the MATLAB. Some parameters in the simulation were set as follows: the time duration was $300 \mathrm{~s}$; the simulation area was $300 \mathrm{~m} \times$ $300 \mathrm{~m}$; the network consists of 50 nodes except the ZC, and all of them were the ZigBee routers; the nodes were randomly deployed following the uniform distribution; $\mathrm{Cm}, \mathrm{Rm}$, and $\mathrm{Lm}$ were set to 4,4 , and 5, separately; the data packet size was 100 bits; the packet interval time was $1 \mathrm{~s}$; the first packet arrival time followed a uniform distribution from 10 to 11 for each node; the maximum retries was four times; the transmitter power of the $\mathrm{ZC}$ is five times the power of other devices; for each scenario, the simulation was carried out 500 times to calculate the average. To make a comprehensive comparison, the two modes of the MPD in which the fOpt was 0 and 1 were both tested. Our algorithm was compared with the two specified routing algorithms, the HRP and the Z-AODV. As a classic method in the hierarchical topology, the EHRP in [29] was also included.

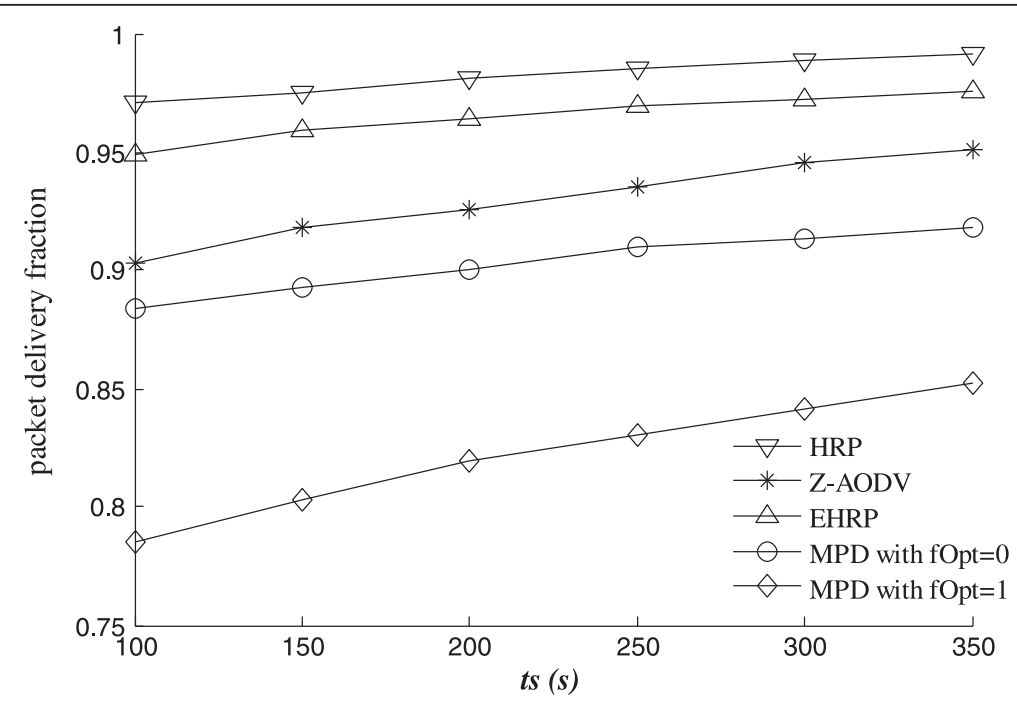

Figure 4 The PDF of different routing algorithms with different mobility. 


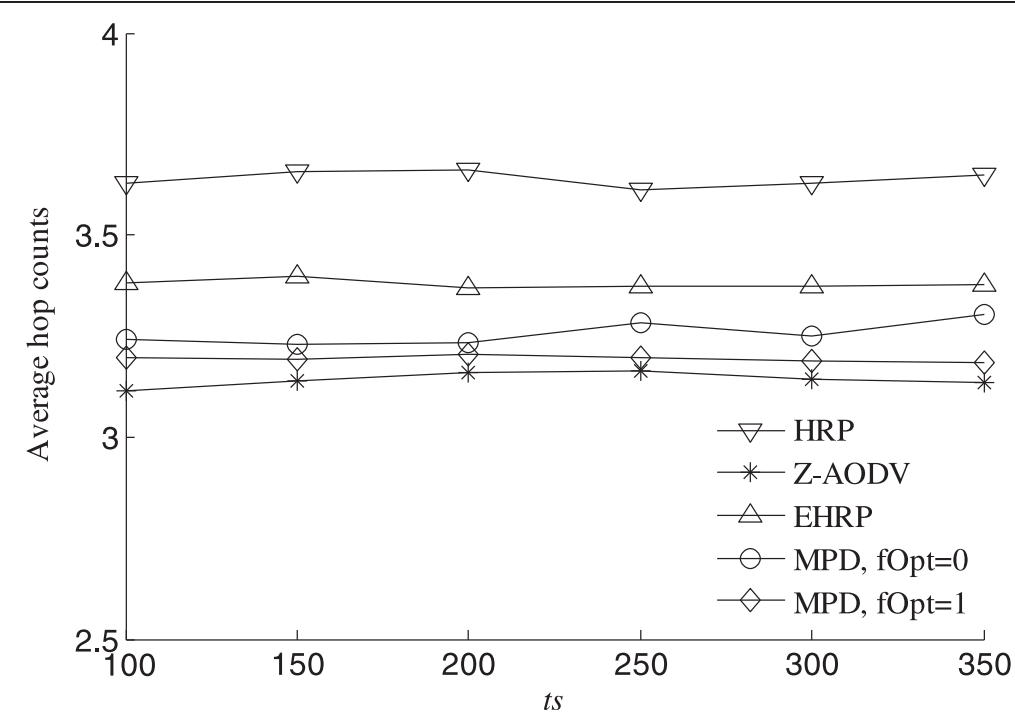

Figure 5 The hop counts of different routing algorithms.

The node mobility should be considered in the wireless networks. In our simulations, each node was stationary for a random time that followed a uniform distribution form ts -50 to ts $+50 \mathrm{~s}$. Then, the node moved to a new position which is randomly chosen, and the moving speed was uniformly distributed from 1 to $10 \mathrm{~m} / \mathrm{s}$. The devices in the smart grid application did not move a lot, so the ts value was ranged from 100 to $350 \mathrm{~s}$. As the duration was $300 \mathrm{~s}$, the ts $=350$ meant all the nodes may not move during the scenario.

We also made a mapping from the LQI in the received data to the signal power. In our simulation, the channel followed Rayleigh fading with $\mathrm{a} \delta^{2}$ of 5 . We mapped the best LQI ( $-3-\mathrm{dB}$ loss) to $0 \times \mathrm{ff}(255)$ and the lowest quality compliant signals detectable by the receiver ( $-20-\mathrm{dB}$ loss) to $0 \times 00(0)$; the values in between were uniformly distributed.

As a protocol based on broadcasting without acknowledgement, we are more concerned with the reliability of the MPD. So the packet delivery fraction (PDF) of different routing methods with different node mobility is discussed firstly. The simulation result is shown in Figure 4. The HRP and EHRP were based on the tree structure and had the best PDF. The routing discovery led to the worse performance of the Z-AODV. For the MPD, when the fOpt was set to 1 , the packet might be transmitted in multiple paths. And it had the worst performance. When the fOpt was 0 , the link was unique, and it had a better result.

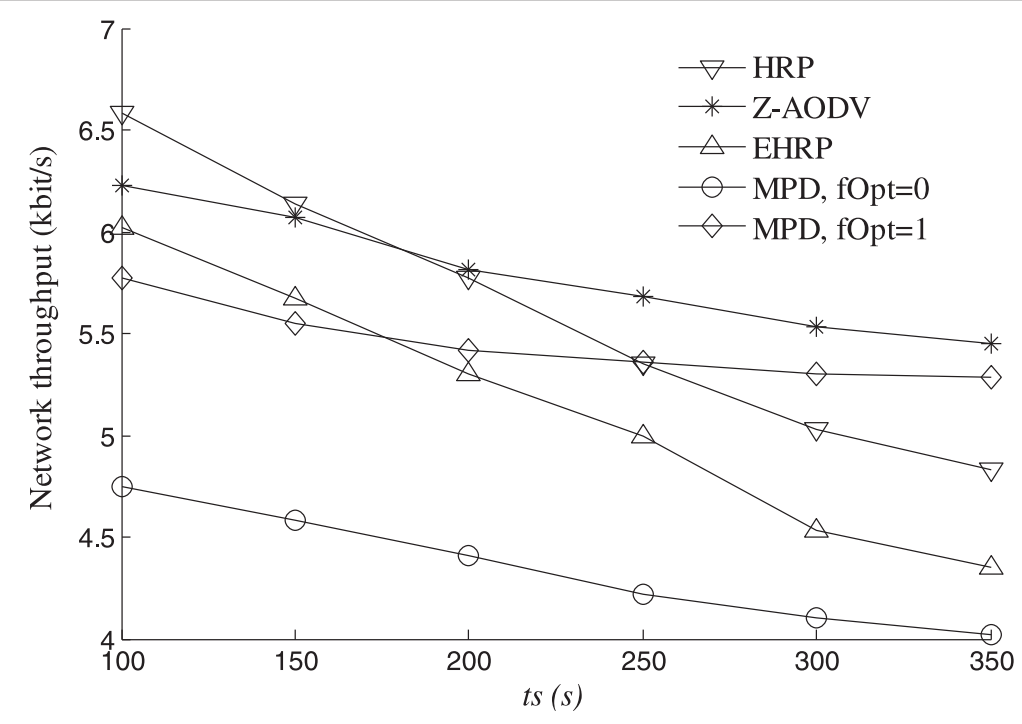

Figure 6 The throughput of different routing algorithms. 


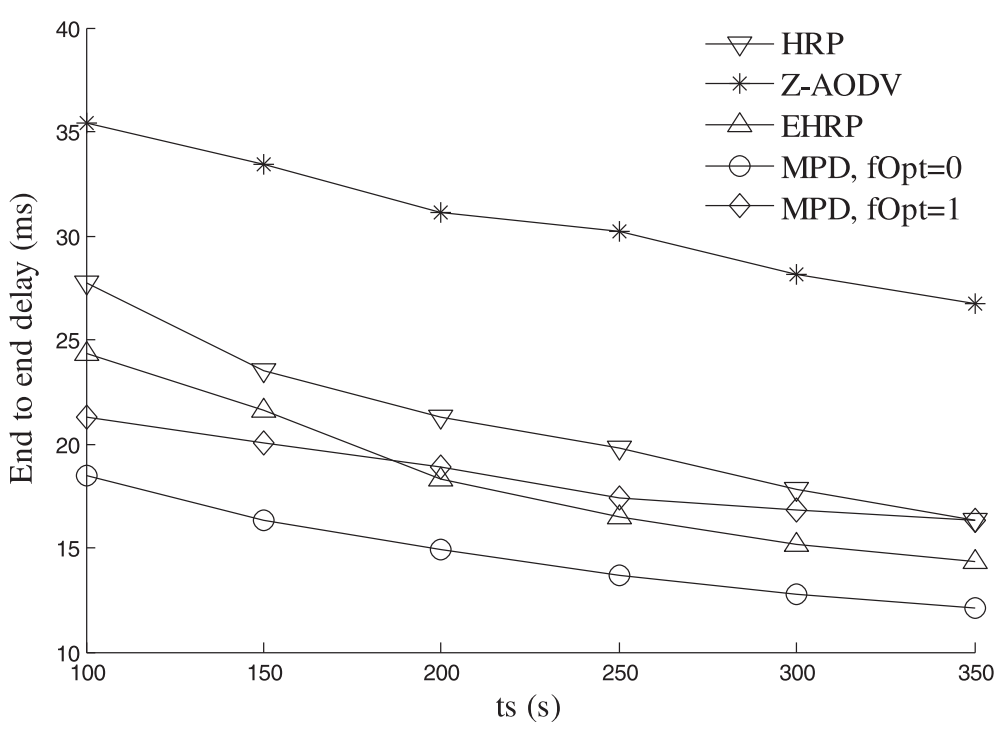

Figure 7 The end-to-end delay in different routing algorithms.

Because our algorithm did not use the acknowledgement, the reliability was declined. However, the performance was acceptable in the wireless network. In the scenarios with the most mobility, the PDF was no less than 0.88 ; in the static network, this value might be more than 0.925 .

The path length obtained by each method is also shown in Figure 5. The HPR had the most hop counts due to the limit of parent-child link, while the Z-AODV could always use the global shortest paths. The EHRP could reduce the path length effectively by using the neighbour table information. We might find that the MPD could further shorten the path, the concept of the PD was similar as the global shortest. However, when one node had more than one neighbour nodes with the same minimum PD, the path was decided only based on the link quality in the current hop, which might lead to the worse performance compared with the Z-AODV. But our algorithm could significantly improve the routing without any routing discovery operations and communication. Moreover, the asymmetric link in the neighbourhood of the ZC was considered for better performance. From the figure, we could conclude that the number of hop counts was less related to the node mobility since each curve did not change much as the ts increased.

Although the routing method is mainly a NWK layer protocol, its performance is not only based on the NWK behaviours but also the MAC schemes. The MPD is quite different from the existing algorithms, even if it is designed for the ZigBee specification, some functions in the IEEE 802.15.4 (e.g. the beacon enable mode, GTS allocation, the superframe structure and etc.) may be imcompatible with our algorithm. It has two meanings; the MPD cannot support such modes. Consequently, the MAC and NWK headers are redundant if we just consider the transmission. To make a meaningful comparison, we did not include all the frame headers in our simulations, only the information related to the routing and the used transmission mechanism was focused on.

Figure 6 illustrates the throughput of different routing methods; the load of the ZC was not included. We could see that the MPD could significantly reduce the network throughput in our simulations. Because the tree structure was rapidly changed, the HRP and EHRP had poor performance in a high mobility network. As an ad hoc protocol, the Z-AODV had a stable but higher performance due to its on-demand routing discovery. When the fOpt was set to 1 , the MPD had similar performance with the existing algorithms, it could be found changed less when the mobility was decreasing. When the fOpt was 0 , the bits in the network were greatly reduced. That is due to the shorter path and also because the response and acknowledgement were not needed.

Similar as the analysis of the network load, the simulation on the end-to-end delay is also a complicated task related to the MAC scheme. Moreover, most simulation software, such as the OPNET, NS2, and NS3, do not provide the broadcasting-based transmission strategy

Table 1 The overall comparison in monitoring and controlling packets transmission

\begin{tabular}{lcccc}
\hline Algorithms & $\begin{array}{c}\text { Packet delivery } \\
\text { fraction }\end{array}$ & $\begin{array}{c}\text { Average hop } \\
\text { counts }\end{array}$ & $\begin{array}{c}\text { Network } \\
\text { throughput }\end{array}$ & Latency \\
\hline HRP & High & More & More & Medium \\
EHRP & High & More & Medium & Medium \\
Z-AODV & Medium & Least & More & High \\
MPD $(f O p t ~=1)$ & Low & Less & Less & Low \\
$M P D(f O p t ~=0)$ & Low & Less & Less & Low \\
\hline
\end{tabular}


used in the MPD. We can only estimate the delays according to the IEEE 802.15.4 protocol. Since the carrier transmission time is so short that can be ignored, the delay is mainly caused by the device processing time and the CSMA/CA mechanism. The former factor was tested in the OPNET as reference, we made a simple network with two nodes (not including the $\mathrm{ZC}$ ) and watched the processing time of the intermediate node with the fixed packet size (100 bit). When trying to send the message, a node may listen to the channel and decide to transmit or wait for a random time based on the CSMA/CA scheme. The simulation in our work is a rough comparison, as a comprehensive work, the accurate test data may be obtained by designing compatible models in our further work.

Shown in Figure 7 is the transmission delay in different routing algorithms. The Z-AODV had the highest latency owing to its routing discovery process. The delay of the HRP was shorter since the transmission was limited in the hierarchical links. And the EHRP could further reduce the delay by shortening the path length. We could see that the latency of the MPD was greatly decreased. Even the fOpt was 1, the delay was similar as the hierarchical routing methods. When the fOpt was 0 , the delay could be obviously reduced. It had two main reasons: one was the shortest path and hop counts, the other was that the bandwidth was saved by removing the receiving acknowledgement and the channel was more likely to be free. Compared with the EHRP, the MPD could improve the performance on the delay for about $19.2 \%$.

The overall comparison is showed in Table 1 . The MPD outperformed the other algorithms in the network throughput and the latency. It also had quite short path length that was only slightly more than the global shortest path in the Z-AODV. The MPD had a low PDF, however, the rate could be more than 0.92 and even in the worst cases we simulated, the PDF was no less than 0.87 with the $f \mathrm{Opt}=0$. Since the setting of $f \mathrm{Opt}=1$ was only used in the first communication, the MPD could be considered reliable. Based on the analysis above, we could conclude that the MPD could effectively improve the data transmission for the monitoring and controlling packets.

\section{Conclusion}

The ZigBee is a fitting communication protocol for the AMI of the smart grid. However, the routing algorithms specified in the ZigBee specification could not fully satisfy the requirements on the energy consumption and the characteristics of the application should be more considered. In this paper, we proposed a minimum physical depth delivery protocol to improve the monitoring and controlling data transmission in the AMI. By introducing the concept of the physical depth, the packet was forwarded along the trajectory of the devices with the least PD in the neighbourhood. Considering the low payload of the frames, the broadcasting mechanism was used to save the bandwidth and the receiving acknowledgement was removed. Our algorithm was compared with the ones specified in the ZigBee and some improved classic methods. The simulation results showed that the MPD could improve the routing performance for the monitoring and controlling packets. It could reduce the network throughput and the hop counts effectively, and the end-to-end delay was slightly decreased. And it had a good-enough packet delivery fraction to meet the requirement of reliability.

Our further work will focus on two issues. One is to design a fully compatible MAC scheme for the minimum physical depth delivery protocol to test the performance more accurately and apply the algorithm in the real application. The other one is to make the protocol aware of the channel quality based on the LQI to avoid the high bit error rate.

\section{Competing interests}

The authors declare that they have no competing interests.

\section{Acknowledgements}

This work is funded by the National Science Foundation of China, (NSFC: 61271411, 61372097), the Project of Tianjin University Technology Development Fund (20130714), and the Doctoral Scientific Foundation of Tianjin Normal University (52XB1106).

Received: 14 May 2014 Accepted: 2 June 2014

Published: 3 July 2014

\section{References}

1. W Sun, X Yuan, J Wang, D Han, C Zhang, Quality of Service Networking for Smart Grid Distribution Monitoring (Paper presented at the IEEE international conference on smart grid communications (SmartGridComm), Gaithersburg, MD, 2010), pp. 373-378. doi:10.1109/SMARTGRID.2010.5622072

2. G Guérard, SB Amor, A Bui, Survey on smart grid modelling. Int. J. Syst. Control Commun. 4(4), 262-279 (2012). doi: 10.1504/IJSCC.2012.050822

3. PP Parikh, MG Kanabar, TS Sidhu, Opportunities and Challenges of Wireless Communication Technologies for Smart Grid Applications (Paper presented at the in power and energy society general meeting, Minneapolis MN, 2010), pp. 1-7. doi:10.1109/PES.2010.5589988

4. Y Zhang, W Sun, L Wang, H Wang, RC Green, M Alam, A Multi-Level Communication Architecture of Smart Grid Based on Congestion Aware Wireless Mesh Network (Paper presented at the IEEE international conference on North American power symposium (NAPS), Boston MA, 2011), pp. 1-6. doi:10.1109/NAPS.2011.6025169

5. P Yi, A Iwayemi, C Zhou, Developing ZigBee deployment guideline under WiFi interference for smart grid applications. IEEE Trans. Smart Grid. 2(1), 110-120 (2011). doi:10.1109/TSG.2010.2091655

6. WP Luan, Advanced metering infrastructure. South Power Syst. Technol. 3(2), 6-10 (2009). http://www.cnki.net/kcms/detail/detail.aspx?dbname=CJFD2009 \&filename=NFDW200902006\&v=MTIzMTZZb1FPRFE4OHZCWm03RXAv VDM2UjJob3hGckNVUkw2ZIIPZHNGeW5tVTc3Skt5dlBIYkcOSHRqTXJZOUY=

7. National Institute of Standards and Technology, Standards identified for inclusion in the smart grid interoperability standards framework. (2009). http://www.nist.gov/smartgrid/upload/NIST_Framework_Release_2-0_corr. pdf. Accessed 20 Dec 2009

8. ZigBee Standard Organization, ZigBee Specification Document $053474 r 17$ (ZigBee Alliance, San Ramon, 2007)

9. Q Liang, L Wang, Event detection in wireless sensor networks using fuzzy logic system, in IEEE International Conference on Computational Intelligence for Homeland Security and Personal Safety, Orlando, 31 Mar - 1 Apr 2005, pp. 52-56. doi: 10.1109/CIHSPS.2005.1500610 
10. H Farhangi, The path of the smart grid. IEEE Power Energy Mag. 8(1), 18-28 (2010)

11. F Cuomo, SD Luna, U Monaco, T Melodia, Routing in ZigBee Benefits from Exploiting the IEEE 802.15.4 Association Tree (Paper presented at the IEEE international conference on communications, Glasgow, 2007), pp. 3271-3276. doi:10.1109//CC.2007.542

12. AA Tareq, SH Yang, A ZigBee-based mobile tracking system through wireless sensor networks. Int. J. Adv. Mechatronic Syst. 1(1), 63-70 (2008). doi:1504/JJAMechS.2008.020839

13. T Metin, K Ibrahim, PSAR: power-source-aware routing in ZigBee networks. Wireless Netw. 18(6), 635-651. doi:10.1007/s11276-012-0424-5

14. Q Ren, Q Liang, Throughput and energy-efficiency-aware protocol for ultrawideband communication in wireless sensor networks: a cross-layer approach. IEEE Trans. Mobile Comput. 7(6), 805-816 (2008)

15. HY Chien, CF Lee, SB Liu, TC Wu, Enhancing convenience and situation-aware intelligence of smart home services using passive RFID and intelligent controls. Int. J. Internet Protoc. Technol. 5(3), 166-173 (2010). doi:10.1504/IJPT.2010.035387

16. Q Liang, Designing power aware self-reconfiguring topology for mobile wireless personal area networks using fuzzy logic. IEEE Trans. Syst. Man. Cybern. C. Appl. Rev. 33(3), 390-394 (2003)

17. P Ran, SM Zou, ZigBee routing Selection Strategy Based on Data Services and Energy-Balanced Zigbee Routing (Paper presented at the IEEE asia-pacific conference services computing, Guangzhou, Guangdong, 2006), pp. 400-404. doi: 10.1109/APSCC.2006.116

18. Z Sahinoglu, P Orlik, J Zhang, B Bhargava, Tree-based data broadcast in IEEE 802.15.4 and ZigBee networks. IEEE Trans. Mobile Comput. 5(11), 1561-1574 (2011)

19. P Ajgaonkar, Simulation studies on ZigBee communications for home automation and networking, in IEEE Autotestcon, Orlando, FL, USA, 13-16 Sept 2010, pp. 1-6. doi:10.1109/AUTEST.2010.5613588

20. Q Ren, Q Liang, Fuzzy logic-optimized secure media access control (FSMAC) protocol wireless sensor networks, in IEEE International Conference on Computational Intelligence for Homeland Security and Personal Safety, Orlando, 31 Mar-1 Apr 2005

21. Q Liang, L Wang, Q Ren, Fault-tolerant and energy efficient cross-layer design for wireless sensor networks. Int. J. Sens. Netw. 2(3), 248-257 (2007)

22. B Lu, TG Habetler, RG Harley, A Novel Motor Energy Monitoring Scheme using Wireless Sensor Networks (Paper presented at the 41st IAS annual meeting industry applications conference, Tampa FL, 2006), pp. 2177-2184. doi:10.10.1109/IAS.2006.256844

23. SW Luan, JH Teng, CS Chen, YH Liu, Development of an Intelligent Fault Indicator for Smart Grids (Paper presented at the international conference on information and electronics engineering, Bangkok Thailand). http://www.ipcsit.com/vol6/5-E011.pdf

24. C Gezer, M Niccolini, C Buratti, An IEEE 802.15.4/ZigBee Based Wireless Sensor Network for Energy Efficient Buildings (Paper presented at the IEEE 6th international conference on wireless and mobile computing, networking and communications, Niagara Falls, ON, Canada, 2010), pp. 486-491. doi:10.1109/WIMOB.2010.5645021

25. SW Luan, JH Teng, LC Hwang, CS Chen, Wireless Network Deployment for Intelligent Fault Indicators - a case study with ZigBee (Paper presented at the IEEE region 10 conference, Bali, 2011), pp. 1246-1250. doi:10.1109/ TENCON.2011.6129006

26. JY Cheng, MH Hung, JW Chang, A ZigBee-Based Power Monitoring System with Direct Load Control Capabilities (Paper presented at the IEEE international conference on networking, sensing and control, London UK, 2007), pp. 15-17. doi:10.1109//AS.2006.256844

27. S Zhang, HL Zhou, An improvement of ZigBee tree routing in the application of intelligent irrigation. ISDEA 1, 255-260 (2010). doi:10.1109/ISDEA.2010.257

28. M Al-Harbawi, MFA Rasid, NK Noordin, Improved tree routing (ImpTR) protocol for ZigBee networks. IJCSNS 9(10), 146-152 (2009). http://paper.ijcsns.org/07_book/200910/20091019.pdf
29. JY Ha, HS Park, S Choi, WH Kwon, EHRP: enhanced hierarchical routing protocol for ZigBee mesh networks. IEEE Commun. Lett. 11(12), 1028-1103 (2007). doi:10.1109/LCOMM.2007.071325

30. T Kim, D Kim, N Park, SE Yoo, Shortcut Tree Routing in ZigBee networks (Paper presented at the second international symposium on wireless pervasive computing, San Juan, 2007), pp. 42-47. doi:10.1109/ ISWPC.2007.342571

31. P Gburzynski, B Kaminska, W Olesinski, A Tiny and Efficient Wireless Ad-Hoc Protocol for Low-Cost Sensor Networks (Paper presented at the conference on design, automation and test in Europe, Nice, 2007), pp. 1-6. doi:10.1109/DATE, 2007.364523

doi:10.1186/1687-1499-2014-108

Cite this article as: Mu: A minimum physical distance delivery protocol based on ZigBee in smart grid. EURASIP Journal on Wireless Communications and Networking 2014 2014:108.

\section{Submit your manuscript to a SpringerOpen ${ }^{\circ}$ journal and benefit from:}

- Convenient online submission

- Rigorous peer review

- Immediate publication on acceptance

- Open access: articles freely available online

- High visibility within the field

- Retaining the copyright to your article

Submit your next manuscript at $\gg$ springeropen.com 